\title{
Transplanted Neural Precursors Enhance Host Brain-Derived Myelin Regeneration
}

\author{
Ofira Einstein, ${ }^{1}$ Yael Friedman-Levi, ${ }^{1}$ Nikolaos Grigoriadis, ${ }^{2}$ and Tamir Ben-Hur ${ }^{1}$ \\ ${ }^{1}$ Department of Neurology, The Agnes Ginges Center for Human Neurogenetics, Hadassah-Hebrew University Medical Center, Jerusalem 91120, Israel, \\ and ${ }^{2} \mathrm{~B}^{\prime}$ Department of Neurology, AHEPA University Hospital of Thessaloniki, 54636 Thessaloniki, Greece
}

In multiple sclerosis lesions resident oligodendrocyte progenitor cells (OPCs) are present, but fail to remyelinate. In the current study we examined whether neural precursor cell (NPC) transplantation can facilitate host brain-derived remyelination. We used the chronic cuprizone-induced demyelination model in aged mice, in which slow remyelination follows cuprizone removal. NPCs were transplanted to the lateral ventricles (intracerebroventricular) of cuprizone-induced demyelinated brains. In this experimental setup, transplanted cells remained mostly in the periventricular area in an undifferentiated state. The extent of demyelination, remyelination, and proliferation of host brain regenerative cell population were examined at 1 week posttransplantation in the splenium of the corpus callosum, which was devoid of any transplanted cells. Transplantation of NPCs, but not of control, human embryonic kidney cells, significantly enhanced remyelination compared with sham-operated mice. Remyelination was performed exclusively by host brain OPCs. The proregenerative effect of transplanted NPCs was related to an increase in the proliferation of host brain OPCs. To examine the mechanism that underlies the proregenerative effect of NPCs in vitro, we used an NPC-OPC coculture system. These experiments indicated that NPCs induced the proliferation of OPCs and facilitated their differentiation into mature oligodendrocytes. The mitogenic effect of NPCs was mediated by platelet-derived growth factor-AA and fibroblast growth factor-2. In conclusion, NPC transplantation enhances hostderived myelin regeneration following chronic demyelination. This trophic effect may stimulate resident OPCs to overcome the remyelination failure in multiple sclerosis.

\section{Introduction}

Multiple sclerosis (MS) is a chronic demyelinating disease of the CNS. Spontaneous remyelination and functional recovery are possible in early stages of disease (Prineas et al., 1993; Raine and Wu, 1993; Compston, 1997; Dubois-Dalcq et al., 2008), but eventually fail (Franklin, 2002). While existing therapies in MS are directed at alleviating the immune attack on the CNS, there are currently no means to improve oligodendrocyte recovery and myelin regeneration. Studies in various experimental models of demyelination have indicated that remyelination is performed by resident oligodendrocyte progenitor cells (OPCs) (Gensert and Goldman, 1997; Keirstead et al., 1998). Successful remyelination depends on migration of OPCs toward the lesion, their proliferation and differentiation into remyelinating oligodendrocytes (Carroll and Jennings, 1994; Redwine and Armstrong, 1998; Keirstead and Blakemore, 1999; Levine et al., 2001). In MS, although resident OPCs are found around the lesions, they remain in a quiescent state and are not recruited sufficiently to the regenerative process (Scolding et al., 1998; Wolswijk, 1998; Maeda et al., 2001; Chang et al., 2002).

Received July 13, 2009; revised 0ct. 19, 2009; accepted 0ct. 26, 2009.

This work was supported by the Schorr Foundation and by the Israel Science Foundation (140/05, T.B.-H.). We thank Maria Chioteli and George Papadopoulos for technical support. We thank Monique Dubois-Dalcq and Bill Blakemore for their valuable comments on the manuscript.

Correspondence should be addressed to Dr. Tamir Ben-Hur, Department of Neurology, Hadassah-Hebrew University Medical Center, Ein-Kerem, P.0. Box 12000, Jerusalem 91120, Israel. E-mail: tamir@hadassah.org.il.

DOI:10.1523/JNEUROSCI.3364-09.2009

Copyright $\odot 2009$ Society for Neuroscience ～0270-6474/09/2915694-09\$15.00/0
Myelin regeneration can be improved either by cell replacement therapy, as a substitute to the endogenous pool of OPCs (Groves et al., 1993; Kocsis, 1999; Zhang and Duncan, 2000), or by boosting the brain's intrinsic capacity for remyelination. While neural precursor cells (NPCs) have promising remyelinating potential (Keirstead et al., 1999), recent studies have suggested that they may also exert neurotrophic properties (Teng et al., 2002; Zhang et al., 2007; Einstein and Ben-Hur, 2008). We therefore hypothesized that NPC transplantation may enhance the myelin regeneration capabilities of host brain cells. To this end we used the model of chronic cuprizone exposure which produces extensive demyelination (Mason et al., 2004; Vana et al., 2007) in aged mice, in which the rate of remyelination is slowed down (Shields et al., 1999; Sim et al., 2002; Shen et al., 2008). We show that intracerebroventricular (ICV) NPC transplantation induced a notable improvement in remyelination in cuprizone-treated mice. The enhanced remyelination was attributed solely to endogenous cells. ICV-transplanted NPCs facilitated host brain OPC proliferation, an effect mediated by platelet-derived growth factor (PDGF)-AA and fibroblast growth factor (FGF)-2. These results suggest that transplanted NPCs exert trophic effects on their environment to enhance remyelination by the host brain pool of progenitor cells.

\section{Materials and Methods}

Animals. C57BL/6 female mice were supplied by Harlan and were grown under specific pathogen-free conditions. Animal experimentation was approved by the institutional Ethics Committee. 


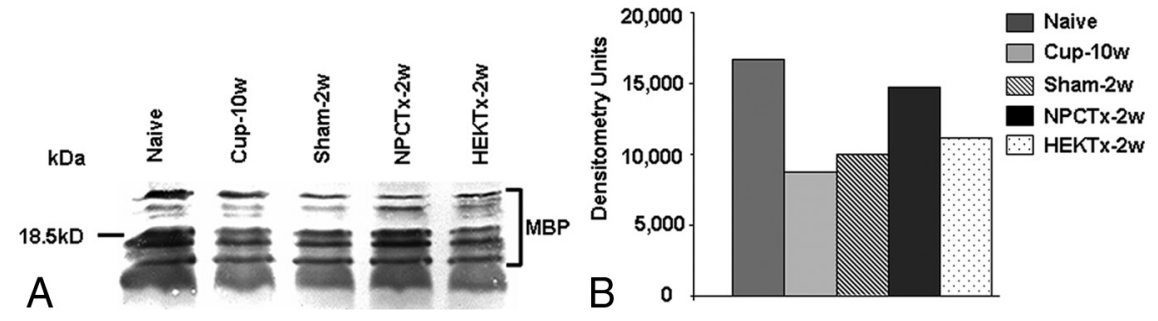

Figure 1. Intracerebroventricular transplantation of NPCs induces an increase in MBP content in the corpus callosum of mice after cuprizone-induced demyelination. A representative (of two experiments) Western blot ( $\boldsymbol{A}$, different bands indicate various isoforms of MBP) and densitometrical semiquantitative analysis $(\boldsymbol{B})$ of myelin basic protein in the corpus callosum of normal (Naive) mice, after a 10 week cuprizone diet (Cup-10w), and in mice exposed to cuprizone at 2 weeks after returning to normal diet in sham-operated (Sham-2w), in NPC-transplanted (NPCTx-2w), and in HEK cell-transplanted mice (HEK-Tx-2w). MBP content was significantly reduced in response to cuprizone diet and slightly elevated following cuprizone withdrawal. NPC, but not HEK cell, transplantation induced a marked elevation in MBP content.

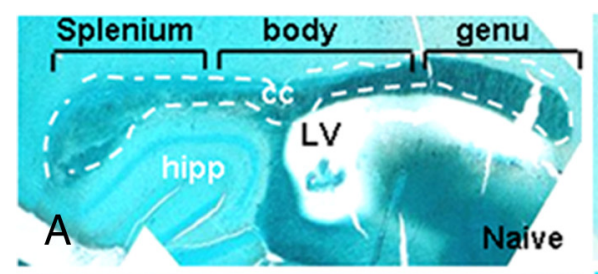

B
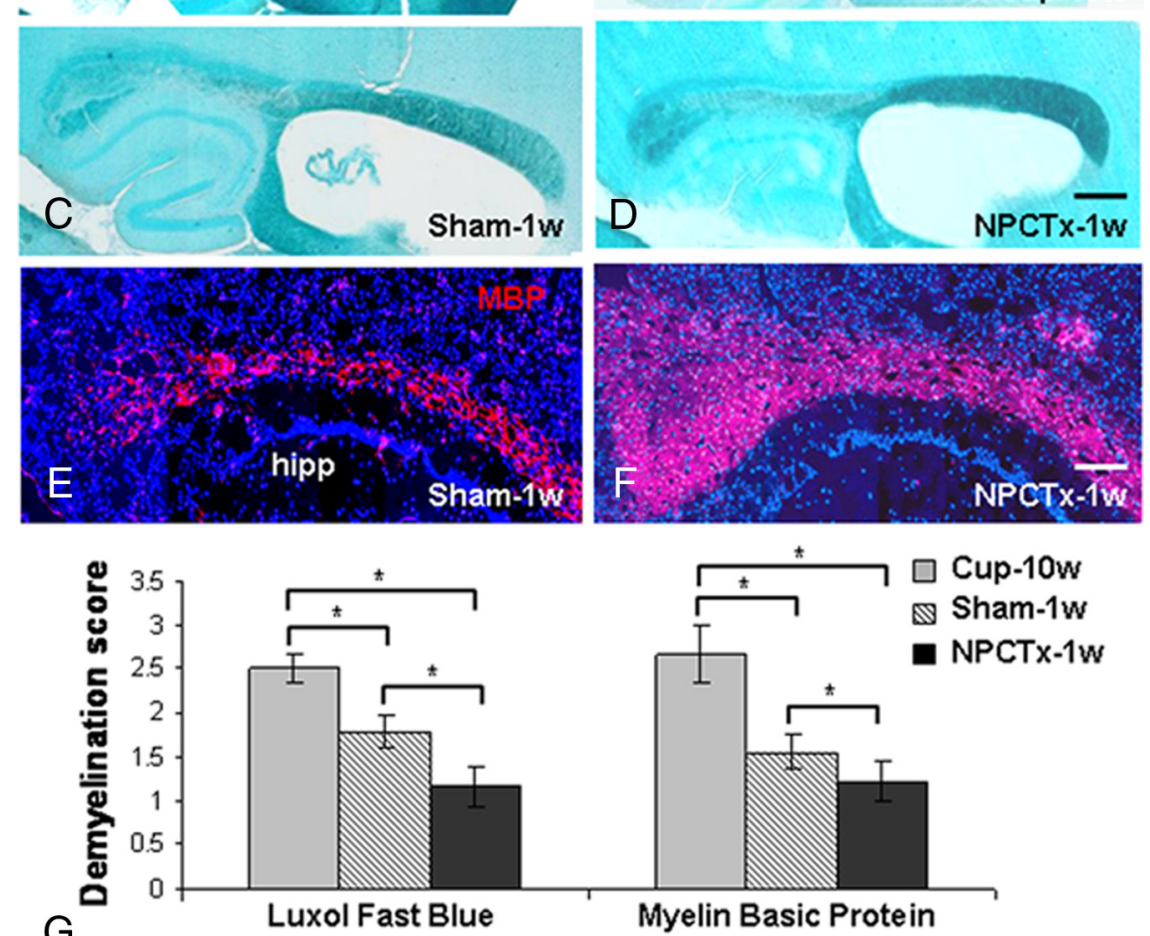

G
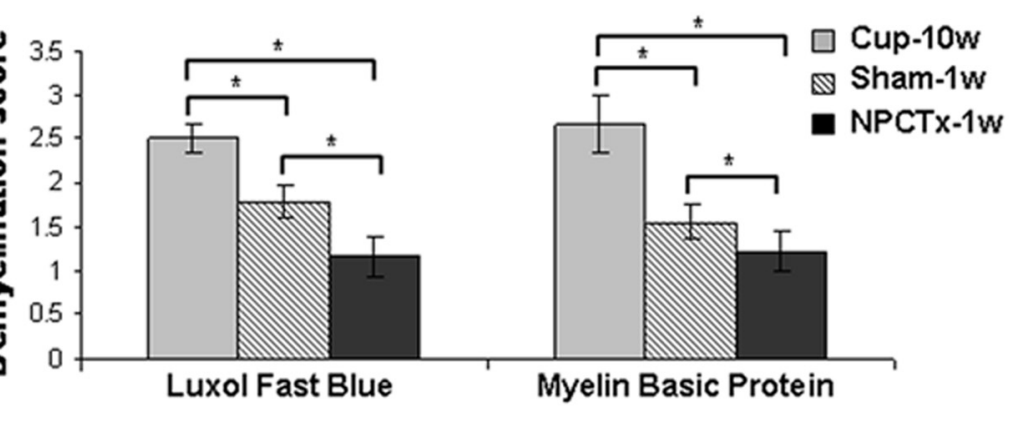

Figure 2. Intracerebroventricular transplantation of NPCs facilitates remyelination in the corpus callosum of mice after cuprizone-induced demyelination. Myelin content was evaluated by luxol fast blue histochemistry $(\boldsymbol{A}-\boldsymbol{D})$ and myelin basic protein immunofluorescent staining ( $\boldsymbol{E}, \boldsymbol{F}$; splenium and body of the corpus callosum). Corpus callosum of a naive mouse ( $\boldsymbol{A}$, delineated by white lines), after a 10 week cuprizone diet (Cup-10w; $\boldsymbol{B}$ ), and in mice exposed to cuprizone at 1 week after returning to normal diet in sham-operated (Sham-1w; $\boldsymbol{C}, \boldsymbol{E})$, and in NPC-transplanted mice (NPCTx-1w; $\boldsymbol{D}, \boldsymbol{F})$. Quantification of myelin content in the splenium $(\boldsymbol{G})$ shows that, within 1 week of returning to normal diet, there was significant decrease in the demyelination score. In NPC-transplanted mice there was a further significant decrease in the demyelination score, compared with sham-operated mice. cc, corpus callosum; hipp, hippocampus; LV, lateral ventricle. Scale bars: (in D) $\boldsymbol{A}-\boldsymbol{D}, 1 \mathrm{~mm}$; (in F) $\boldsymbol{E}, \boldsymbol{F}, 250 \mu \mathrm{m}$. Data are represented as mean $\pm \mathrm{SEM},{ }^{*} p<0.05$.

Growth of mouse NPC spheres. Multipotential NPCs were isolated from newborn transgenic C57BL/6 mice expressing green fluorescent protein (GFP; obtained from Japan SLC), as previously described (Einstein et al., 2006b).
Cuprizone-induced experimental chronic demyelination. In preliminary experiments we defined the optimal dose of cuprizone (biscyclohexane oxaldihydrazone, Sigma-Aldrich) to be used for achieving chronic demyelination in 5- to 6-month-old female C57BL/6 mice. A dose of $0.3 \% \mathrm{w} / \mathrm{w}$ cuprizone in a diet of ground mouse chow for a period of 10 weeks was then used $(n=30)$. After 10 weeks of cuprizone diet, mice were killed for Western blot analysis $(n=3)$ or for histological analysis $(n=6)$ and the rest were switched back to normal chow. Some mice of the same age were fed with their normal diet to serve as naive, nontreated controls $(n=9)$.

Cell transplantation and bromodeoxyuridine injection. ICV transplantation was performed on the day of switching back to the normal diet as previously described (Einstein et al., 2006b). Quantities of $2.5 \times 10^{3} \mathrm{GFP}+\mathrm{NPC}$ spheres $(n=9)$, or $2.5 \times 10^{5}$ human embryonic kidney cells (HEK293; obtained from ATCC, $n=3$ ), in a volume of $5 \mu \mathrm{l}$ or $5 \mu \mathrm{l}$ of F12/DMEM (sham-operated; $n=9$ ) were injected once into each lateral ventricle. For identifying proliferating cells in the brain, NPC-transplanted $(n=3)$ and sham-operated $(n=3)$ mice were injected intraperitoneally with bromodeoxyuridine (BrdU) $(50 \mu \mathrm{g} / 1 \mathrm{~g}$ of body weight, Sigma-Aldrich), from the day of transplantation for 7 consecutive days.

Preparation of brain homogenates and immunoblotting. Naive mice and mice 2 weeks after ICV transplantation ( $n=3$ /group) were decapitated for Western blot analysis. A midline longitudinal section of the brain was performed to separate the cerebral hemispheres. The cerebellum, brainstem, and cortices were removed under a dissecting microscope and the entire corpus callosum was isolated. Tissue slices containing the corpus callosum were homogenized to $10 \%(\mathrm{w} / \mathrm{v})$ in cold homogenization buffer ( $320 \mathrm{~mm}$ sucrose; $10 \mathrm{~mm}$ EDTA in PBS), followed by centrifugation at $3000 \mathrm{rpm}$ for $15 \mathrm{~min}$ at $4^{\circ} \mathrm{C}$. Homogenates from each group were pooled and normalized for total protein level using a BCA assay (Pierce); $50 \mu \mathrm{l}$ from each sample were extracted with $2 \%$ Sarkosyl, applied to 15\% SDS-PAGE, and electrotransferred to nitrocellulose membrane in a Tris/glycine buffer. Membranes were blocked with $5 \%$ milk in TBST (10 mm Tris- $\mathrm{HCl}, \mathrm{pH}$ $7.4,150 \mathrm{~mm} \mathrm{NaCl}, 0.05 \%$ Tween 20 ), incubated overnight at $4^{\circ} \mathrm{C}$ with rat anti-myelin basic protein (MBP; MAB 386, Millipore Bioscience Research Reagents, 1:1000) or with mouse anti-prion protein (PrP; IPC1, P5999, SigmaAldrich, 1:5000, served as control) primary antibodies, washed and incubated for $1 \mathrm{~h}$ with anti-rat or anti-mouse AP secondary antibodies (Promega 1:7000). The relative intensities of the bands were measured by NIH ImageJ analysis software. The control PrP level was similar in all groups.

Tissue processing for histopathological analyses. One week after ICV injection, mice $(n=$ $6 /$ group) were perfused with $4 \%$ paraformaldehyde (PFA, Fluka) for histochemistry and immunofluorescence stainings ( $n=3$ /group), or with $2 \%$ PFA and $0.5 \%$ glutaraldehyde (GLA, Fluka) for resin embedding ( $n=3$ /group). Naive, untreated 
mice were also perfused with $4 \%$ PFA $(n=3)$ or $2 \%$ PFA and $0.5 \%$ GLA $(n=3)$.

Histopathology. Serial $8 \mu \mathrm{m}$ longitudinal frozen sections were cut laterally between midline and bregma $1.8 \mathrm{~mm}$. Sections were stained for myelin with luxol fast blue (LFB; Sigma-Aldrich) and examined by light microscopy. For immunofluorescent staining, sections and cultures (fixated with $2 \%$ PFA) were incubated with the following primary antibodies: rat anti-MBP (MAB 386, Millipore Bioscience Research Reagents, 1:100), rabbit IgG anti-NG2 (Millipore Bioscience Research Reagents, 1:50), rat anti-BrdU (OBT0030G, Clone BU1/75(ICR1), Serotec, 1:200), goat anti-Olig2 (AF2418, R\&D Systems, 1:100), mouse IgM antiPDGFR $\alpha$ (R\&D Systems, 1:20), rabbit anti-glial fibrillary acidic protein (GFAP; Dako, 1:100), mouse IgM anti-O4 (Millipore Bioscience Research Reagents, 1:50), rabbit anti-galactocerebroside (GalC; Millipore Bioscience Research Reagents, 1:30), as previously described (Einstein et al., 2006b). The following secondary antibodies were used where appropriate: Alexa 555- or Alexa 488-conjugated goat anti-rabbit IgG (Invitrogen, 1:200), goat anti-rat (Invitrogen, 1:200), donkey anti-goat IgG (Invitrogen, 1:200), goat anti-mouse IgM (Invitrogen, 1:200), and mounted with DAPI (4', $6^{\prime}$-diamidino-2-phenylindole dihydrochloride) (Vector Laboratories) for nuclei staining. Immunostained sections were examined by a fluorescent microscope.

PFA- and GLA-fixated brains were cut longitudinally to separate the two hemispheres. One hemisphere was trimmed to leave a tissue block that contained the entire corpus callosum, and then $50 \mu \mathrm{m}$ longitudinal vibratome slices were cut laterally around the transplantation site $(0.85-$ 1.35 lateral to bregma). To exclude the presence of transplanted cells in areas where semithin sections were performed, $50 \mu \mathrm{m}$ slices were visualized under a fluorescent microscope for GFP + cells before further resin processing. Resin blocks were prepared at the caudal end of the splenium of the corpus callosum, which did not contain any GFP+ cells. Sections $(1 \mu \mathrm{m})$ were cut at $0.95-1.05$ lateral to bregma, stained with $1 \%$ toluidine blue (Sigma-Aldrich), and examined by light microscopy.

Biodistribution and differentiation of transplanted NPCs. Transplanted NPCs were identified by fluorescence microscopy for GFP expression on 3 nonadjacent longitudinal $8 \mu \mathrm{m}$ frozen sections, representing the entire corpus callosum. These sections were also stained for NG2, Olig2, GalC, MBP, and GFAP expression, as described above.

Quantification of de-and remyelination in the corpus callosum. Evaluation of myelin content was performed on three nonadjacent LFB- or MBP-stained frozen sections, representing the entire corpus callosum. The extent of demyelination in the splenium of the corpus callosum was scored in a blinded manner, using a standard scale (Lindner et al., 2008): 0 , normal myelin; 1 , one third of myelin loss; 2 , two thirds of myelin loss; and 3, complete demyelination, as is the norm.

Detailed evaluation of myelin integrity in the splenium of corpus callosum was performed on three randomly chosen toluidine blue-stained semithin sections ( $n=3$ /group). Each section was visualized under fixed illumination of a light microscope (at $100 \times$ magnification) and three microscopic fields per section were stored on a computer using a video camera. At least 300 fibers per animal were analyzed, using the ImageJ software, and their $G$ ratios were calculated ( $G=$ axon diameter/(axon + myelin sheath diameter)). A nerve fiber with $G$ ratio $>0.8$ was considered remyelinated. The percentage of normally myelinated, de- and remyelinated axons from the total number of axons was calculated.

Quantification of OPC proliferation in the corpus callosum. Cells were counted in three nonadjacent $8 \mu \mathrm{m}$ longitudinal frozen sections per brain ( $n=3$ /group). Quantification of cells was performed specifically in three regions of the corpus callosum: splenium, body, and genu. NG2+ or $\mathrm{NG} 2+, \mathrm{BrdU}+$ or Olig2 + cells were counted under a fluorescence microscope, using a $40 \times$ objective and a grid overlay. Average cell densities were calculated as number of cells per $\mathrm{mm}^{2}$.

Cocultures of OPCs and NPC spheres. OPCs were enriched from 7-dold mixed glial cultures by the shaking method, as previously described (Ben-Hur et al., 1998), and then plated on $10 \mu \mathrm{g} / \mathrm{ml}$ poly-D-lysine (PDL, Sigma-Aldrich)-coated glass coverslips in 24-well dishes (Costar, Corning), at a density of $1 \times 10^{5}$ cells per well in $1 \mathrm{ml}$ of modified $\mathrm{N} 2$ medium. At plating these cultures consisted of $>95 \% \mathrm{NG} 2+$ cells. In some wells 3000 NPC spheres (to form an NPC:OPC ratio of 3:1) were added in the

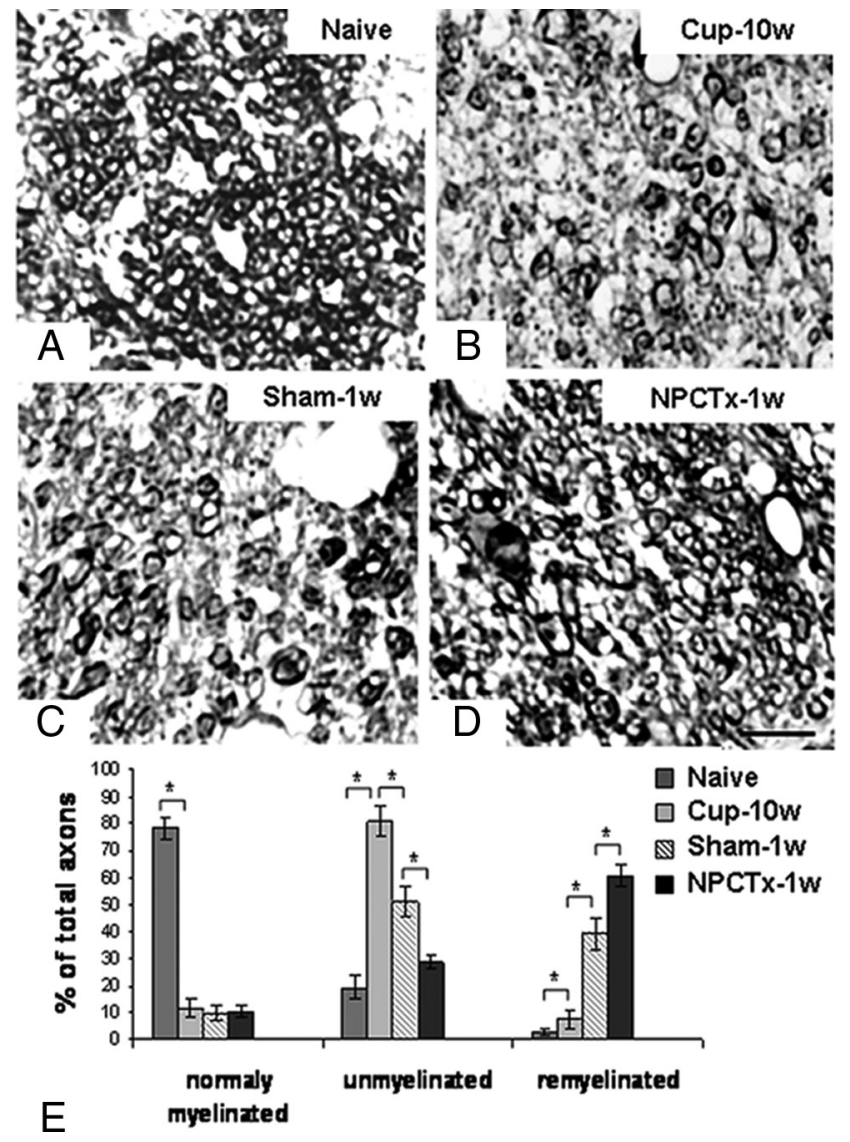

Figure 3. Intracerebroventricular transplantation of NPCs facilitates remyelination: highresolution analysis in the splenium. Remyelination in the splenium of the corpus callosum was evaluated on toluidine blue-stained semithin sections of naive mice $(\boldsymbol{A})$, after a 10 week cuprizone diet (Cup-10w; $\boldsymbol{B}$ ), and in mice exposed to cuprizone at 1 week after returning to normal diet in sham-operated (Sham-1w; ) and in NPC-transplanted (NPCTx-1w; $\boldsymbol{D}$ ) mice. In naive mice there were mainly normal axons $(\boldsymbol{A}, \boldsymbol{E})$, whereas after the 10 week cuprizone diet there were mainly demyelinated axons $(\boldsymbol{B}, \boldsymbol{E})$. One week after returning to normal diet, there was a significant rise in the fraction of remyelinated axons in sham-operated mice $(\boldsymbol{C}, \boldsymbol{E})$. In NPCtransplanted mice there was a significantly higher fraction of remyelinated axons $(\boldsymbol{D}, \boldsymbol{E})$. Scale bar: (in $\boldsymbol{D}) \boldsymbol{A}-\boldsymbol{D}, 5 \mu \mathrm{m}$. Data are represented as mean $\pm \mathrm{SEM},{ }^{*} p<0.05$.

upper chamber of a Transwell insert system, separated from the OPCs in the lower chamber by a semipermeable membrane (Costar, Corning). In some wells $10 \mathrm{ng} / \mathrm{ml}$ human FGF2 (R\&D Systems), or $10 \mathrm{ng} / \mathrm{ml}$ human PDGF-AA (R\&D Systems) or their combination were added daily. Some wells of OPC-NPC sphere cocultures were incubated in the presence of the neutralizing antibodies mouse IgG anti-bovine FGF2 $(50 \mu \mathrm{g} / \mathrm{ml}$, clone bFM-1, Upstate) or goat IgG anti-human PDGF-AA $(20 \mu \mathrm{g} / \mathrm{ml}$, Upstate) or their combination. The cultures were examined for the presence of proliferating OPCs after $3 \mathrm{~d}$, and for oligodendrocytes after $5 \mathrm{~d}$ in culture. For labeling proliferating OPCs, the cultures were pulsed with 20 $\mu \mathrm{M}$ BrdU during the last $24 \mathrm{~h}$. Then NPC spheres were discarded from the upper chamber, and the adherent cells on the coverslips from the lower chamber were double-immunostained for BrdU and NG2 or PDGFR $\alpha$. Differentiation and maturation of oligodendrocytes was evaluated after $5 \mathrm{~d}$ in culture by immunostaining for $\mathrm{O} 4$ and GalC. Stained cells were visualized under a fluorescent microscope at $20 \times$ magnification. $\mathrm{NG} 2+$, $\mathrm{BrdU}+$ cells or $\mathrm{PDGFR} \alpha+,+\mathrm{BrdU}+$ cells, or $\mathrm{O} 4+$ or GalC + cells were counted in 3 randomly chosen fields per coverslip. O4+ cell branching was measured by counting the number of pixels of stained area per cell, using Adobe PhotoShop software. All individual culture conditions were analyzed in triplicates.

Statistical analysis. Data from several experiments were pooled to obtain the mean \pm SE (SEM) for each experimental group. For histological parameters or for coculture experiments, the experimental groups and 

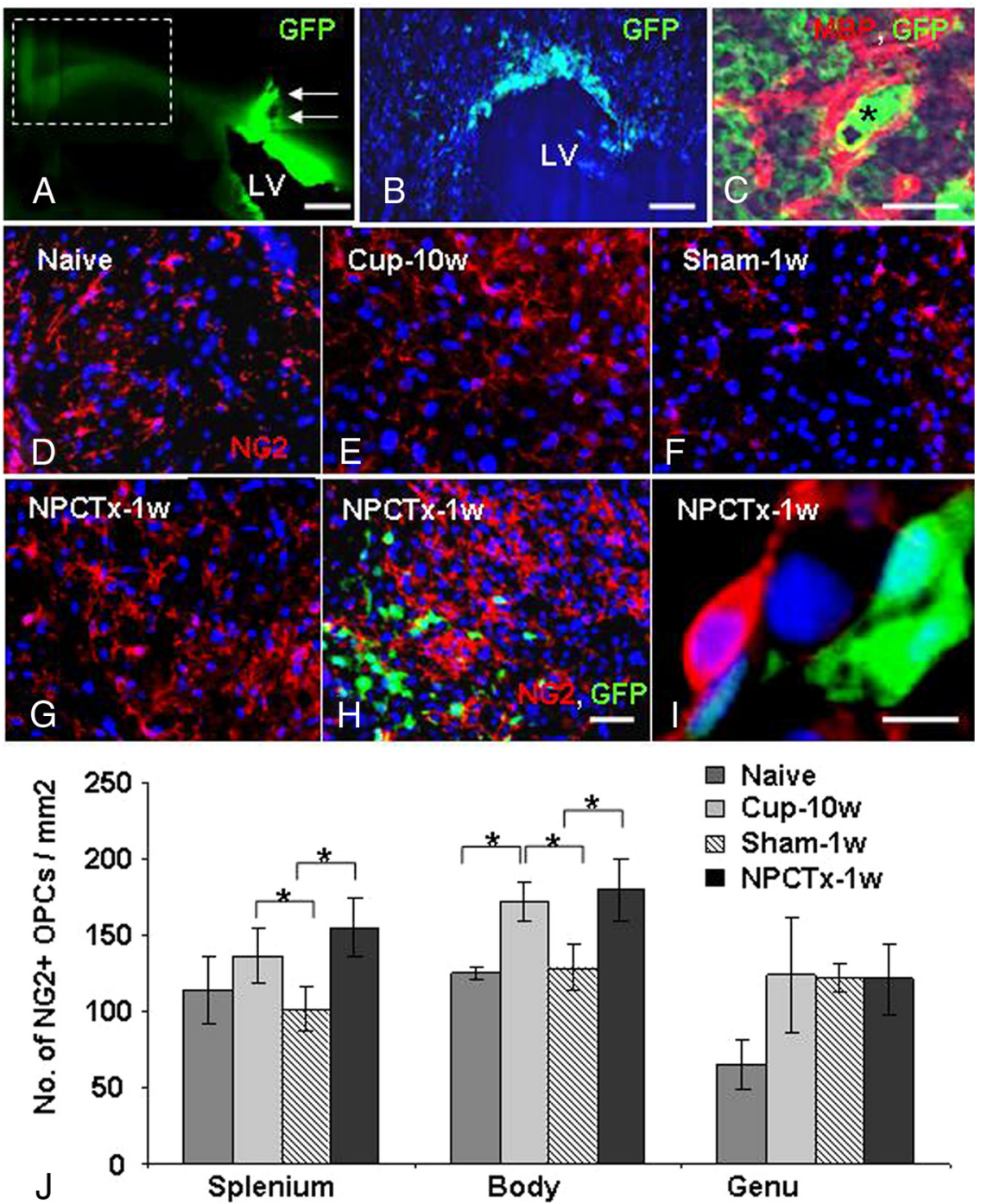

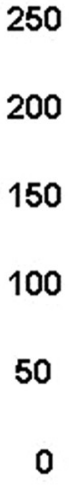

0

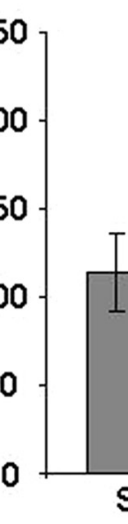

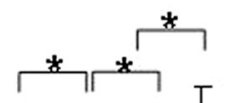

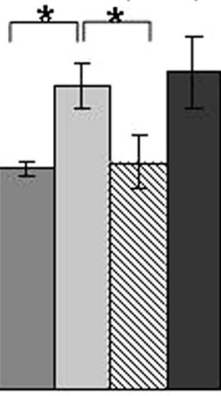

Body
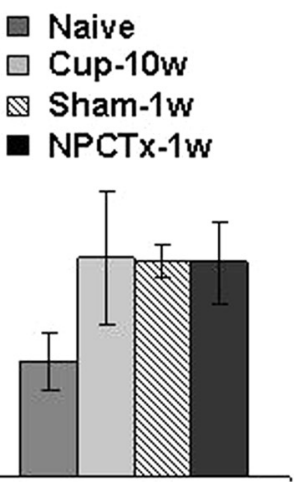

Genu

Figure 4. NPC transplantation enhances the repopulation of chronic cuprizone-induced demyelinated lesions by host brainderived oligodendrocyte progenitor cells. Low-power field fluorescence microscopy ( $\boldsymbol{A}$, arrows; needle tract), followed by highpower field microscopy $(\boldsymbol{B})$ indicated that at 1 week posttransplantation the GFP $+N P C$ s remained mainly in the periventricular area and did not reach the splenium of the corpus callosum. Confocal microscopy of GFP transgenic mouse brain indicated that MBP + oligodendrocytes coexpress GFP (C; GFP, green; MBP, red; asterisk indicates location of nucleus according to dark-field microscopy). NG2 + OPCs (red) were detected in the splenium of the corpus callosum of naive mice $(\boldsymbol{D})$ after 10 weeks of cuprizone exposure (Cup-10w; $\boldsymbol{E}$ ), in mice exposed to cuprizone at 1 week after cuprizone withdrawal in sham-operated mice $($ Sham-1w; $\boldsymbol{F})$, and in NPC-transplanted mice (NPCTx-1w; G). GFP + cells had penetrated up to few cell layers in the periventricular area $(\boldsymbol{H})$, but not further in the white matter. No GFP + cells were observed in the splenium, where all NG2 + OPCs were host brain-derived (G). Also in the body of the corpus callosum the vast majority of OPCs were GFP- host brain cells $(\boldsymbol{H}, \boldsymbol{I})$. OPC numbers increased significantly after 10 weeks' cuprizone exposure and returned to baseline levels within 1 week after returning to normal diet in sham-operated mice $(\boldsymbol{J})$. In NPC-transplanted mice there was a further increase in resident GFP - , NG2 + OPC number in both the body and splenium (J). Scale bars: $\boldsymbol{A}, 1 \mathrm{~mm} ; \boldsymbol{B}, 250 \mu \mathrm{m} ; \boldsymbol{C}, \boldsymbol{I}, 10 \mu \mathrm{m} ; \boldsymbol{D}-\boldsymbol{H}$ (in $\boldsymbol{H}$ ), $100 \mu \mathrm{m}$. Data are represented as mean \pm SEM, ${ }^{*} p<0.05$

control groups were compared using one-tailed Student's $t$ test. Differences were considered significant at $p<0.05$.

\section{Results}

Chronic cuprizone exposure in aged mice provides a relevant model to characterize the repair capacity of endogenous cells Prolonged feeding with cuprizone induces widespread demyelination in the corpus callosum of C57BL/6 mice, which can be reversed upon switching back to a normal diet. In advanced age the process of remyelination process is slowed down (Shields et al., 1999; Sim et al., 2002; Shen et al., 2008). In our experiments,

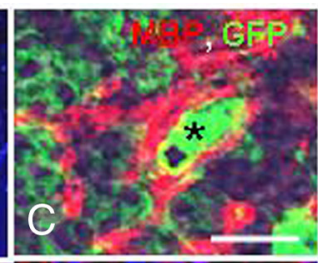

after 10 weeks of $0.3 \%$ cuprizone diet, mice exhibited marked myelin loss, as was indicated by Western blot analysis (Fig. 1) and by LFB histochemistry (Fig. $2 A, B)$. In agreement with previous studies, demyelination was most extensive in the splenium (Stidworthy et al., 2004; Irvine and Blakemore, 2008), less so in the body, and minimal in the genu of the corpus callosum. Two weeks after cuprizone was removed from the diet, partial remyelination was observed, as indicated by an increase in the amount of myelin in the corpus callosum (Fig. 1).

\section{NPC transplantation enhances remyelination}

Using the chronic cuprizone model for demyelination, we examined the effect of NPC transplantation on myelin content. At the end of the 10-week cuprizone diet, a group of mice was transplanted ICV with NPC spheres, derived from newborn syngeneic C57BL/6 mice brains, and killed 2 weeks later for evaluation of myelin content, compared with a group of cuprizone-treated, HEK-transplanted or sham-operated mice. Myelin loss in the corpus callosum was evaluated by Western blot analysis for MBP (Fig. 1). In control, sham-operated mice MBP content in the corpus callosum increased by $12 \%$ within 2 weeks after returning to a normal diet (Fig. 1). In NPC-transplanted animals there was a further $48 \%$ increase in MBP content, compared with shamoperated controls, and reaching a level comparable to that of naive animals (Fig. 1). In HEK-transplanted C57BL/6 mice brains there was no increase in MBP content when compared with sham-operated mice (Fig. 1)

Given the fact that NPC transplantation induced almost complete remyelination in the corpus callosum of cuprizone-treated mice at 2 weeks after cuprizone withdrawal and cell transplantation, we turned to examine the effects of NPC transplantation at an earlier time point, during the dynamic phase of remyelination. Myelin content was therefore examined at 1 week after NPC or sham transplantation by image analysis of LFB- and MBP-stained sections (Fig. $2 C-G$ ). In sham-operated mice the demyelination score in the splenium was reduced by $28 \%$ by LFB staining ( $p=$ 0.004 ), and by $41 \%$ by MBP staining; (Fig. $2 G ; p=0.004$ ). In NPCtransplanted animals there was a greater increase in myelin content than in sham-operated controls, as indicated by $52 \%$ and $56 \%$ reduction in the demyelination score, by LFB and MBP stainings, respectively (Fig. $2 G$ ). Thus, NPC transplantation induced within 1 week a $34 \%$ (by LFB; Fig. $2 C, D, G ; p=0.05$ ) or $25 \%$ (by MBP; Fig. $2 E-G ; p=0.02$ ) enhancement in remyelination. 
For detailed evaluation of remyelination we identified normally myelinated, demyelinated and remyelinated axons by calculating the G-ratio of individual axons in toluidine blue-stained semithin sections (Fig. 3A-E). In naive mice there were $78.2 \pm 4 \%$ myelinated axons and $19.3 \pm 4 \%$ nude axons, with very few axons corresponding to the definition of remyelinated axons (Fig. $3 A, E$ ). After 10 weeks of cuprizone diet there was an $85 \%$ reduction (to $11.7 \pm 3 \%$ ) in normally myelinated axons (Fig. $3 B, E ; p<0.001$ ) and a $77 \%$ increase in demyelinated axons (to $80.6 \pm 5 \%, p<0.001)$. Ten weeks of cuprizone diet were associated with some remyelinating process, as indicated by the presence of $7.6 \pm 3.5 \%$ remyelinated fibers. At 1 week after switching to normal diet a spontaneous remyelinating process was evident (Fig. 3C,E) in sham-operated mice, as indicated by the presence of $39.2 \pm 6 \%$ remyelinated axons $(p<0.001$, compared with 10 week cuprizone group), and a reduction in the fraction of demyelinated axons to $51 \pm 5 \%(p<0.001)$. In NPC-transplanted mice the remyelination process was significantly enhanced, as indicated by additional $43 \%$ reduction in demyelinated axons (to $28.7 \pm 2 \%$, Fig. $3 D, E ; p<0.001)$ and $55 \%$ increase in remyelinated axons (to $60.7 \pm 4 \%, p<$ 0.001 ), compared with sham-operated mice. Thus, NPC transplantation facilitated remyelination in the corpus callosum of C57BL/6 mice following removal of cuprizone from the diet.

\section{Distribution and fate of transplanted NPCs}

To define the contribution of host brain cells versus that of transplanted cells to the process of remyelination, the distribution and differentiation of transplanted cells in the brains of cuprizone-exposed C57BL/6 mice was studied. Transplanted newbornderived NPCs were detected in the host brain by their GFP fluorescence. The bulk of the graft cells were attached to the ventricular wall and along the needle tract (Fig. 4A). Detailed microscopy showed that there was very limited migration of transplanted cells into the caudal border of the body of the corpus callosum (Fig. $4 B$ ) and no GFP+ NPCs were detected in the splenium, where quantification of demyelination and remyelination was performed. Immunofluorescent staining for NG2, Olig2, MBP, GalC, and GFAP indicated that the vast majority of transplanted GFP + NPCs remained in an undifferentiated state (data not shown). Specifically, $<1 \%$ of transplanted GFP + cells were NG2 + OPCs, and these were restricted solely to the periventricular area. Moreover, there was no further migration or differentiation of transplanted GFP + cells, when examined in brains at 2 weeks posttransplantation. Notably, to rule out the possibility that NPC differentiation into mature oli-
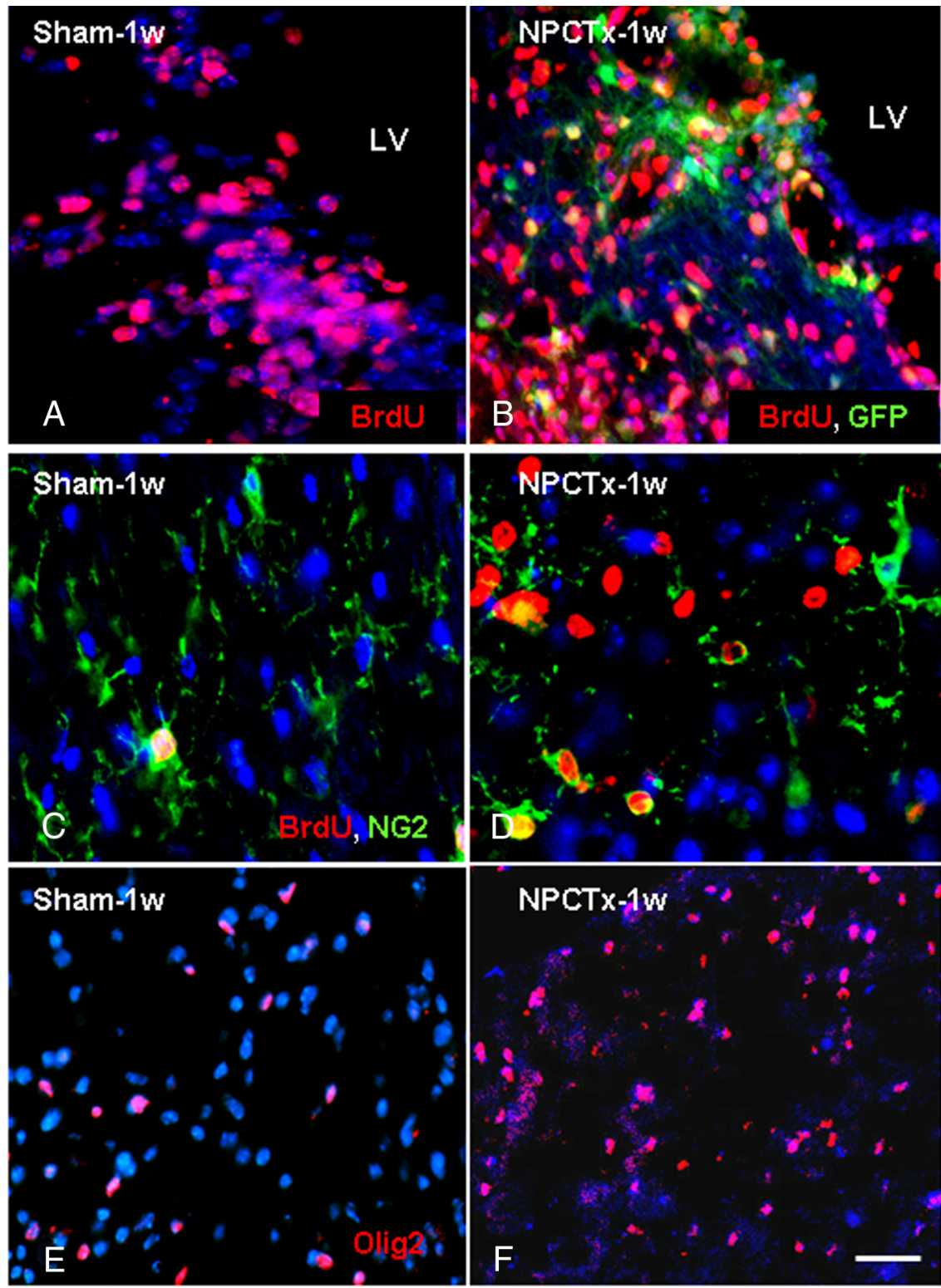

Figure 5. NPC transplantation enhances the proliferation of host brain-derived oligodendrocyte lineage cells in chronic cuprizone-induced demyelinated lesions. $\operatorname{BrdU}+$ cells were detected (red) in the body $(\boldsymbol{A}, \boldsymbol{B})$ and in the splenium $(\boldsymbol{C}, \boldsymbol{D})$ of the corpus callosum in mice exposed to cuprizone at 1 week after cuprizone withdrawal in sham-operated $(\boldsymbol{A}, \boldsymbol{C})$ and NPC-transplanted $\boldsymbol{B}, \boldsymbol{D})$ mice. The density of BrdU + cells increased in the vicinity of transplanted GFP $+N P C$ in the body of the corpus callosum $(\boldsymbol{B})$, were no grafted cells, BrdU + (red), NG2 + (green) cell density increased significantly in NPC-transplanted mice (D), compared with sham-operated mice (C). The density of Olig2 + cells (red) was significantly increased in the splenium of transplanted mice $(\boldsymbol{G})$, compared with sham-operated mice $(\boldsymbol{F})$. Scale bar: $100 \mu \mathrm{m}$. Data are represented as mean $\pm \mathrm{SEM},{ }^{*} p<0.05$.

godendrocytes is not associated with loss of GFP+ fluorescence, frozen sections from GFP transgenic mice were stained for MBP. Detailed confocal microscopy evaluation of brain white matter tracts (e.g., corpus callosum, internal capsule, cerebellar peduncles) indicated that MBP staining colocalized with GFP fluorescence (Fig. $4 C$ ). Thus, the enhanced remyelinating process in the splenium of the corpus callosum of transplanted animals was caused exclusively by host brain cells.

\section{Trophic effect of transplanted NPCs involves induction of} endogenous OPC proliferation

We next examined the effect of transplantation on the density of remyelinating cells in vivo. NG2+ OPCs were quantified in the 

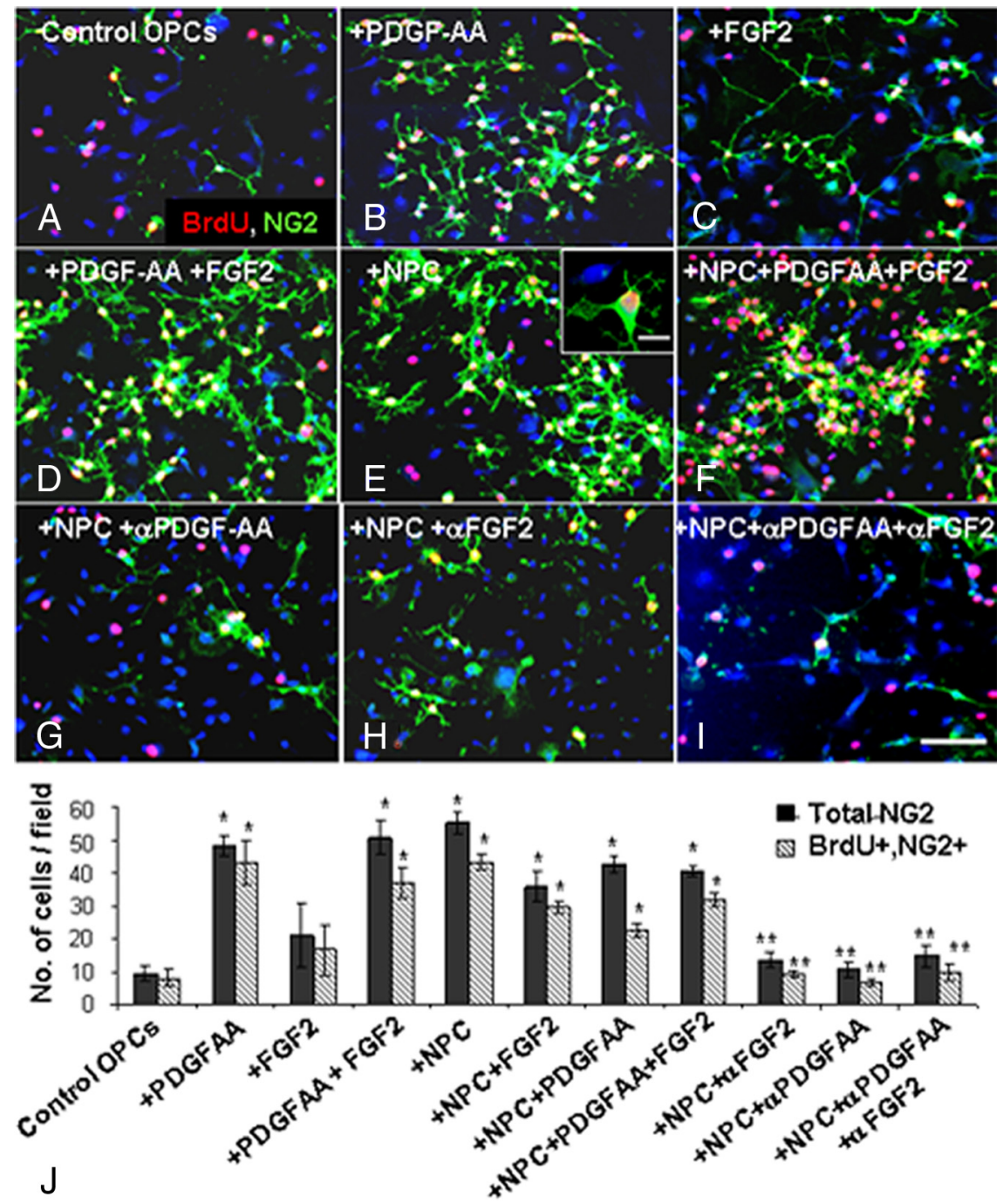

Figure 6. NPCs induce the proliferation of oligodendrocyte progenitor cells in vitro. Purified OPC cultures were incubated in various conditions and pulsed with BrdU to identify proliferating cells ( $A-I, N G 2$, green; BrdU, red; DAPI, blue). The total number of $\mathrm{NG} 2+$ cells and NG2 +, BrdU + cells per microscopic field was quantified $(\boldsymbol{J})$. As compared with control nontreated cultures $(\boldsymbol{A})$, the addition of PDGF-AA $(\boldsymbol{B})$ or FGF2 $(\boldsymbol{C})$, or both $(\boldsymbol{D})$ significantly increased the number of proliferating OPCS $(\boldsymbol{J})$. In cocultures, where NPCs were placed in the upper chamber of a Transwell system, a comparable increase in OPC proliferation was observed $(\boldsymbol{E}, \boldsymbol{J})$. Addition of PDGF-AA and FGF2 to the coculture had no additional effect on OPC proliferation $(\boldsymbol{F}, \boldsymbol{J})$. Addition of anti-PDGF-AA $(\boldsymbol{G})$ or anti-FGF2 $(\boldsymbol{H})$ neutralizing antibodies, or both antibodies $(\boldsymbol{I})$ to the cocultures completely blocked the proliferative effect of NPCs on OPCS $(\boldsymbol{J})$. Scale bar: $\boldsymbol{A}-\boldsymbol{I}, 100 \mu \mathrm{m}$; inset in $\boldsymbol{E}, 10 \mu \mathrm{m}$. Data are represented as mean \pm SEM, ${ }^{*} p<$ 0.05 , compared with control, nontreated OPC culture; ${ }^{* *} p<0.05$, compared with OPC $-\mathrm{NPC}$ coculture.

different parts of the corpus callosum of naive C57BL/6 mice (Fig. $4 D, J$ ), after 10 weeks of the cuprizone diet (Fig. $4 E, J$ ), and in cuprizone-treated mice 1 week after reverting to a normal diet with sham (Fig. $4 F, J$ ) or NPC (Fig. $4 G-J$ ) graft. Cuprizone induced a $28-48 \%$ increase in OPCs in different parts of the corpus callosum, as previously reported (Hiremath et al., 1998). OPC numbers decreased to baseline within 1 week after reverting to normal diet in sham-transplanted mice (Fig. $4 J$ ). However, in NPC transplanted mice OPC numbers remained consistently elevated, and were increased, by $35 \%$ ( $p=0.033$ ) in the splenium and $29 \%(p=0.045)$ in the body of the corpus callosum, compared with sham-operated mice (Fig. $4 J)$ ). Interestingly, OPC concentration was highest in the periventricular zone, near the GFP+ grafts, but distinct from GFP+ cells (Fig. $4 H, I$ ), with a gradual decline in their density in the splenium. However, the increase in NG2+ OPC density in transplanted mice was statistically significant even in the distant caudal splenium (Fig. 4G).
TotalNG2 $\mathrm{BrdU}+, \mathrm{NG} 2+$

To examine whether the increase in OPC density in response to NPC transplantation was due to enhanced proliferation or inhibition of OPC differentiation BrdU was injected systemically during the week after cuprizone withdrawal to identify proliferating cells in the brain. Compared with sham-operated mice (Fig. $5 A$ ), a $23 \%$ increase in $\mathrm{BrdU}+$, GFP - cells (from $486.7 \pm 66$ to $630 \pm 65$ cells $/ \mathrm{mm}^{2}$; $p=0.028)$ was detected in the body of corpus callosum of transplanted mice (Fig. 5B). A similar increase was observed in the splenium of corpus callosum, which was completely deficient in transplanted cells. Double immunostaining for BrdU and NG2 indicated that $>80 \%$ of NG2+ OPCs were BrdU+ cycling cells. Accordingly, NPC transplantation induced a $39 \%$ increase in the density of NG2+, BrdU + cells in the splenium of corpus callosum (from $78.3 \pm 12$ to $127.5 \pm 15$ cells $/ \mathrm{mm}^{2} ; p=0.006$; Fig. $5 C, D$ ).

The basic helix-loop-helix transcription factor Olig2 is crucial for OPC maturation into myelinating oligodendrocytes (Zhou et al., 2000; Lu et al., 2002). We therefore characterized the population of cells exhibiting strong Olig2 expression in the recovery period after cuprizone exposure. Compared with sham-operated mice (Fig. 5E), in transplanted mice (Fig. $5 F$ ) there was a $35 \%$ increase in Olig2 + cell density (from $180.6 \pm$ 46 to $274.5 \pm 17$ cells $/ \mathrm{mm}^{2} ; p<0.001$ ) in the splenium of the corpus callosum. Together, these results show that NPC transplantation induced a significant increase in remyelinating cell proliferation during the regenerative phase.

The proregenerative effect of NPCs is mediated by the growth factors PDGF-

\section{$\mathrm{AA}$ and FGF2}

To examine the molecular mechanism underlying the NPC-induced enhanced remyelination in vivo, we used an NPCOPC coculture Transwell system in vitro. NPCs and OPCs were obtained from newborn C57BL/6 mice brains. Specifically, we examined the effect of NPCs (compared with that of PDGF-AA and FGF2) on OPC proliferation and on the production of differentiating oligodendrocytes. In control OPC cultures, grown for $3 \mathrm{~d}$ in the absence of any exogenous growth factor, only $8 \pm$ $2.6 \%$ of the cells were $\mathrm{NG} 2+$, BrdU + cells (Fig. $6 A, J$ ). PDGF-AA induced a fivefold increase in $\mathrm{NG} 2+, \mathrm{BrdU}+$ cell numbers (Fig. $6 B, J ; p=0.001)$, whereas FGF2 induced a nonsignificant twofold increase (Fig. $6 C, J ; p=0.09$ ). The combination of PDGF-AA and FGF2 had no additive effect over PDGF-AA alone (Fig. $6 D, J$ ). In cocultures, where NPCs were cultured in the upper chamber of a Transwell system, separated by a semipermeable membrane from OPCs that were cultured in the lower chamber, there was a 5.5fold increase in OPC proliferation, without the addition of any exogenous growth factor (Fig. $6 E, J ; p<0.001$ ). Similarly, addition of PDGF-AA or coculturing with NPCs induced a threefold 
$(p=0.002)$ increase in the number of PDGFR $\alpha+$ OPCs and a fourfold $(p=$ 0.001 ) increase in the number of BrdU+ OPCs (data not shown). Addition of PDGF-AA or FGF2 or both to OPC-NPC cocultures did not have any additive effect on the NPC-induced proliferation of OPCs (Fig. 6F,J). These results indicate that the mitogenic effects of PDGF-AA and FGF2 on OPCs can be replaced by culturing with NPCs. When neutralizing antibodies to PDGF-AA (Fig. 6G) or to FGF2 (Fig. $6 H$ ) or their combination (Fig. $6 I$ ) were added to the NPC-OPC cocultures there was complete inhibition of OPC proliferation (Fig. $6 \mathrm{~J}$ ). Thus, the mitogenic effect of NPCs is mediated mainly by PDGF-AA and FGF2. Importantly, in all culture conditions BrdU was present in $>90 \%$ of NG2+ cells (Fig. $6 J$ ). This suggests that the NPCs affect OPC by a mitogenic effect that increases OPC proliferation rather than affecting their survival.

Since FGF2 has been shown to suppress OPC differentiation (McKinnon et al., 1990; Murtie et al., 2005), we examined whether NPCs affect OPC differentiation. After $5 \mathrm{~d}$ of culture, the differentiation of OPCs into mature $\mathrm{O} 4+$ or $\mathrm{GalC}+$ oligodendrocytes was compared between the different experimental groups (Fig. $7 A-H$ ). In cultures in which FGF2 was added for the entire $5 \mathrm{~d}$ period (Fig. $7 B$ ) there was a nonsignificant $1.6(p=0.07)$ - and $1.2(p=0.5)$-fold increase in the numbers of $\mathrm{O} 4+$ and $\mathrm{GalC}+$, compared with control, nontreated OPCs (Fig. 7A, G,H). PDGF-AA (Fig. 7C), and the combination of PDGF-AA and FGF2 (Fig. 7D) induced a significant $5(p<0.001)$ - and $7(p<0.001)$-fold increase in $\mathrm{O} 4+$ cell numbers, respectively (Fig. $7 G)$, and $4.2(p<0.001)$ and $4.3(p<0.001)$-fold increase in GalC + numbers, respectively (Fig. 7H). NPC spheres, cocultured with OPCs in a Transwell system (Fig. $7 E)$, induced a significant $6.4(p<0.001)$ - and 3.5 ( $p=0.006)$-fold increase in $\mathrm{O} 4+$ and $\mathrm{GalC}+$ oligodendrocyte numbers, respectively, compared with control, nontreated OPCs (Fig. 7G,H). Thus, coculturing with NPCs promotes the generation of oligodendrocytes from OPCs. In addition, image analysis was performed to evaluate the arborization of oligodendrocytes as an indication of their maturation (data not shown). PDGF-AA induced a twofold increase in $\mathrm{O} 4+$ cell branching. compared with control cultures. Coculturing of OPCs with NPCs induced a threefold increase in branching of $\mathrm{O} 4+$ cells. Together, these data indicate that NPC-induced OPC proliferation at the early time point is mediated by PDGF-AA and FGF2, and that NPCs then facilitate the maturation of OPCs into oligodendrocytes.

\section{Discussion}

This work is the first report to show a trophic effect of transplanted NPCs, to enhance remyelination by host brain cells. Facilitation of remyelination was mediated by a graft-induced increase in host brain OPC proliferation. To demonstrate that, we took advantage of the fact that chronic cuprizone exposure (Mason et al., 2004; Vana et al., 2007) and the use of aged C57BL/6 mice (Shields et al., 1999; Sim et al., 2002; Shen et al., 2008) slows down the rate of spontaneous remyelination. Under these conditions we could examine the effect of NPC transplantation on the proliferation of host brain progenitor cells and on remyelination. Imperative conditions for this experimental set-up were that transplanted newborn C57BL/6 mice-derived NPCs did not migrate into the demyelinated corpus callosum, did not differentiate into oligodendrocyte lineage cells and did not participate directly in the remyelinating process. These findings are consistent with other reports where transplanted NPCs remained in an undifferentiated form (Copray et al., 2006) and that migration and remyelination by transplanted cells are dependent on removal of endogenous progenitors by X-irradiation and on a local inflammatory process (Ben-Hur et al., 2003; Foote and Blakemore, 2005).

As OPC proliferation is a prerequisite for remyelination (Carroll and Jennings, 1994; Redwine and Armstrong, 1998; Keirstead and Blakemore, 1999; Levine et al., 2001), we examined the effect of transplanted NPCs on cycling OPCs in the host C57BL/6 mouse brain. Cuprizone exposure induced amplification in the OPC pool, which returned to the normal brain baseline state within 1 week after cuprizone removal. In contrast, NPC transplantation induced a further increase in the pool of proliferating OPCs. NPCs have been shown to produce a myriad of growth factors (Lu et al., 2003; Einstein et al., 2006a). To identify the factors which mediate NPCs effects on OPCs, both derived form C57BL/6 newborn mice brains, we used a twochamber Transwell coculture system. The finding that NPCs induced a dramatic increase in the proliferation of OPCs, which was completely blocked by neutralizing antibodies to PDGF-AA and FGF2, indicates a crucial role of these factors (and mainly by PDGF-AA) in the mitogenic effect of NPCs. The observed gradient in the number of proliferating endogenous OPCs between the body of the corpus callosum, adjacent to the graft and the splenium, is consistent with an NPC effect that is mediated by such diffusible factors. Indeed, PDGF-AA and FGF2 are key factors for OPC proliferation and for oligodendrogenesis in normal devel- 
opment (Bögler et al., 1990; McKinnon et al., 1990; Wolswijk and Noble, 1992; Calver et al., 1998) and in response to demyelination (Frost et al., 2003; Woodruff et al., 2004; Murtie et al., 2005; Vana et al., 2007). Specifically, FGF2 and PDGF-AA have been shown to transform slow cycling adult OPCs into neonatal-like, rapidly proliferating OPCs (Wolswijk and Noble, 1992). Furthermore, differentiation and myelination by wild type subventricular zone cells grafted in shiverer mice was dependent on concurrent injection of PDGF and FGF (Lachapelle et al., 2002). This may underlie the proregenerative effect of transplanted NPCs on cuprizoneexposed aged mice. Notably, FGF2 has been shown to inhibit OPC differentiation (McKinnon et al., 1990; Murtie et al., 2005; Armstrong et al., 2006). However, in addition to their mitogenic effect, NPCs enhanced maturation of oligodendrocytes in vitro and facilitated remyelination in vivo.

These observations may be relevant for regenerative processes in MS. In many MS lesions immature OPCs and premyelinating oligodendrocytes are present in relatively normal density but do not remyelinate efficiently (Scolding et al., 1998; Wolswijk, 1998; Maeda et al., 2001; Chang et al., 2002). Failure of remyelination was explained in part by a temporal mismatch, in which the resident pool of OPCs does not respond in time to regenerative cues that are present only during a narrow time window (Foote and Blakemore, 2005). Our data suggest that NPC transplantation is a potent method to activate the proliferation of endogenous OPCs and to drive their maturation into remyelinating oligodendrocytes. Remyelination depends not only on available OPCs, but also on tissue support. It is therefore possible that the NPC graft affects also other host tissue elements to enhance remyelination. For example, transplanted NPCs may modulate the functions of reactive astrocytes to support remyelination or downregulate inhibitors of oligodendrogenesis, such as bone morphogenic proteins (Mekki-Dauriac et al., 2002). Thus, the trophic effect of transplanted NPCs may overcome the temporal mismatch and lead to enhancement of endogenous remyelination.

\section{References}

Armstrong RC, Le TQ, Flint NC, Vana AC, Zhou YX (2006) Endogenous cell repair of chronic demyelination. J Neuropathol Exp Neurol 65:245-256.

Ben-Hur T, Rogister B, Murray K, Rougon G, Dubois-Dalcq M (1998) Growth and fate of PSA-NCAM+ precursors of the postnatal brain. J Neurosci 18:5777-5788.

Ben-Hur T, Einstein O, Mizrachi-Kol R, Ben-Menachem O, Reinhartz E, Karussis D, Abramsky O (2003) Transplanted multipotential neural precursor cells migrate into the inflamed white matter in response to experimental autoimmune encephalomyelitis. Glia 41:73-80.

Bögler O, Wren D, Barnett SC, Land H, Noble M (1990) Cooperation between two growth factors promotes extended self-renewal and inhibits differentiation of oligodendrocyte-type-2 astrocyte (O-2A) progenitor cells. Proc Natl Acad Sci U S A 87:6368-6372.

Calver AR, Hall AC, Yu WP, Walsh FS, Heath JK, Betsholtz C, Richardson WD (1998) Oligodendrocyte population dynamics and the role of PDGF in vivo. Neuron 20:869-882.

Carroll WM, Jennings AR (1994) Early recruitment of oligodendrocyte precursors in CNS demyelination. Brain 117:563-578.

Chang A, Tourtellotte WW, Rudick R, Trapp BD (2002) Premyelinating oligodendrocytes in chronic lesions of multiple sclerosis. N Engl J Med 346:165-173.

Compston A (1997) Remyelination in multiple sclerosis: a challenge for therapy. The 1996 European Charcot Foundation Lecture. Mult Scler 3:51-70.

Copray S, Balasubramaniyan V, Levenga J, de Bruijn J, Liem R, Boddeke E (2006) Olig2 overexpression induces the in vitro differentiation of neural stem cells into mature oligodendrocytes. Stem Cells 24:1001-1010.

Dubois-Dalcq M, Williams A, Stadelmann C, Stankoff B, Zalc B, Lubetzki C
(2008) From fish to man: understanding endogenous remyelination in central nervous system demyelinating diseases. Brain 131:1686-1700.

Einstein O, Ben-Hur T (2008) The changing face of neural stem cell therapy in neurologic diseases. Arch Neurol 65:452-456.

Einstein O, Ben-Menachem-Tzidon O, Mizrachi-Kol R, Reinhartz E, Grigoriadis $\mathrm{N}$, Ben-Hur T (2006a) Survival of neural precursor cells in growth factor-poor environment: Implications for transplantation in chronic disease. Glia 53:449-455.

Einstein O, Grigoriadis N, Mizrachi-Kol R, Reinhartz E, Polyzoidou E, Lavon I, Milonas I, Karussis D, Abramsky O, Ben-Hur T (2006b) Transplanted neural precursor cells reduce brain inflammation to attenuate chronic experimental autoimmune encephalomyelitis. Exp Neurol 198:275-284.

Foote AK, Blakemore WF (2005) Inflammation stimulates remyelination in areas of chronic demyelination. Brain 128:528-539.

Franklin RJ (2002) Why does remyelination fail in multiple sclerosis? Nat Rev Neurosci 3:705-714.

Frost EE, Nielsen JA, Le TQ, Armstrong RC (2003) PDGF and FGF2 regulate oligodendrocyte progenitor responses to demyelination. J Neurobiol 54:457-472.

Gensert JM, Goldman JE (1997) Endogenous progenitors remyelinate demyelinated axons in the adult CNS. Neuron 19:197-203.

Groves AK, Barnett SC, Franklin RJ, Crang AJ, Mayer M, Blakemore WF, Noble M (1993) Repair of demyelinated lesions by transplantation of purified O-2A progenitor cells. Nature 362:453-455.

Hiremath MM, Saito Y, Knapp GW, Ting JP, Suzuki K, Matsushima GK (1998) Microglial/macrophage accumulation during cuprizone-induced demyelination in C57BL/6 mice. J Neuroimmunol 92:38-49.

Irvine KA, Blakemore WF (2008) Remyelination protects axons from demyelination-associated axon degeneration. Brain 131:1464-1477.

Keirstead HS, Blakemore WF (1999) The role of oligodendrocytes and oligodendrocyte progenitors in CNS remyelination. Adv Exp Med Biol 468:183-197.

Keirstead HS, Levine JM, Blakemore WF (1998) Response of the oligodendrocyte progenitor cell population (defined by NG2 labelling) to demyelination of the adult spinal cord. Glia 22:161-170.

Keirstead HS, Ben-Hur T, Rogister B, O'Leary MT, Dubois-Dalcq M, Blakemore WF (1999) Polysialylated neural cell adhesion molecule-positive CNS precursors generate both oligodendrocytes and Schwann cells to remyelinate the CNS after transplantation. J Neurosci 19:7529-7536.

Kocsis JD (1999) Restoration of function by glial cell transplantation into demyelinated spinal cord. J Neurotrauma 16:695-703.

Lachapelle F, Avellana-Adalid V, Nait-Oumesmar B, Baron-Van Evercooren A (2002) Fibroblast growth factor-2 (FGF-2) and platelet-derived growth factor $\mathrm{AB}(\mathrm{PDGF}(\mathrm{AB}))$ promote adult SVZ-derived oligodendrogenesis in vivo. Mol Cell Neurosci 20:390-403.

Levine JM, Reynolds R, Fawcett JW (2001) The oligodendrocyte precursor cell in health and disease. Trends Neurosci 24:39-47.

Lindner M, Heine S, Haastert K, Garde N, Fokuhl J, Linsmeier F, Grothe C, Baumgärtner W, Stangel M (2008) Sequential myelin protein expression during remyelination reveals fast and efficient repair after central nervous system demyelination. Neuropathol Appl Neurobiol 34:105-114.

Lu P, Jones LL, Snyder EY, Tuszynski MH (2003) Neural stem cells constitutively secrete neurotrophic factors and promote extensive host axonal growth after spinal cord injury. Exp Neurol 181:115-129.

Lu QR, Sun T, Zhu Z, Ma N, Garcia M, Stiles CD, Rowitch DH (2002) Common developmental requirement for Olig function indicates a motor neuron/oligodendrocyte connection. Cell 109:75-86.

Maeda Y, Solanky M, Menonna J, Chapin J, Li W, Dowling P (2001) Platelet-derived growth factor-alpha receptor-positive oligodendroglia are frequent in multiple sclerosis lesions. Ann Neurol 49:776-785.

Mason JL, Toews A, Hostettler JD, Morell P, Suzuki K, Goldman JE, Matsushima GK (2004) Oligodendrocytes and progenitors become progressively depleted within chronically demyelinated lesions. Am J Pathol 164:1673-1682.

McKinnon RD, Matsui T, Dubois-Dalcq M, Aaronson SA (1990) FGF modulates the PDGF-driven pathway of oligodendrocyte development. Neuron 5:603-614.

Mekki-Dauriac S, Agius E, Kan P, Cochard P (2002) Bone morphogenetic proteins negatively control oligodendrocyte precursor specification in the chick spinal cord. Development 129:5117-5130.

Murtie JC, Zhou YX, Le TQ, Vana AC, Armstrong RC (2005) PDGF and FGF2 pathways regulate distinct oligodendrocyte lineage responses in 
experimental demyelination with spontaneous remyelination. Neurobiol Dis 19:171-182.

Prineas JW, Barnard RO, Kwon EE, Sharer LR, Cho ES (1993) Multiple sclerosis: remyelination of nascent lesions. Ann Neurol 33:137-151.

Raine CS, Wu E (1993) Multiple sclerosis: remyelination in acute lesions. J Neuropathol Exp Neurol 52:199-204.

Redwine JM, Armstrong RC (1998) In vivo proliferation of oligodendrocyte progenitors expressing PDGFalphaR during early remyelination. J Neurobiol 37:413-428.

Scolding N, Franklin R, Stevens S, Heldin CH, Compston A, Newcombe J (1998) Oligodendrocyte progenitors are present in the normal adult human CNS and in the lesions of multiple sclerosis. Brain 121:2221-2228.

Shen S, Sandoval J, Swiss VA, Li J, Dupree J, Franklin RJ, Casaccia-Bonnefil P (2008) Age-dependent epigenetic control of differentiation inhibitors is critical for remyelination efficiency. Nat Neurosci 11:1024-1034.

Shields SA, Gilson JM, Blakemore WF, Franklin RJ (1999) Remyelination occurs as extensively but more slowly in old rats compared to young rats following gliotoxin-induced CNS demyelination. Glia 28:77-83.

Sim FJ, Zhao C, Penderis J, Franklin RJ (2002) The age-related decrease in CNS remyelination efficiency is attributable to an impairment of both oligodendrocyte progenitor recruitment and differentiation. J Neurosci 22:2451-2459.

Stidworthy MF, Genoud S, Li WW, Leone DP, Mantei N, Suter U, Franklin RJ (2004) Notchl and Jagged 1 are expressed after CNS demyelination, but are not a major rate-determining factor during remyelination. Brain 127:1928-1941.
Teng YD, Lavik EB, Qu X, Park KI, Ourednik J, Zurakowski D, Langer R, Snyder EY (2002) Functional recovery following traumatic spinal cord injury mediated by a unique polymer scaffold seeded with neural stem cells. Proc Natl Acad Sci U S A 99:3024-3029.

Vana AC, Flint NC, Harwood NE, Le TQ, Fruttiger M, Armstrong RC (2007) Platelet-derived growth factor promotes repair of chronically demyelinated white matter. J Neuropathol Exp Neurol 66:975-988.

Wolswijk G (1998) Chronic stage multiple sclerosis lesions contain a relatively quiescent population of oligodendrocyte precursor cells. J Neurosci 18:601-609.

Wolswijk G, Noble M (1992) Cooperation between PDGF and FGF converts slowly dividing O-2Aadult progenitor cells to rapidly dividing cells with characteristics of O-2Aperinatal progenitor cells. J Cell Biol 118:889-900.

Woodruff RH, Fruttiger M, Richardson WD, Franklin RJ (2004) Plateletderived growth factor regulates oligodendrocyte progenitor numbers in adult CNS and their response following CNS demyelination. Mol Cell Neurosci 25:252-262.

Zhang SC, Duncan ID (2000) Remyelination and restoration of axonal function by glial cell transplantation. Prog Brain Res 127:515-533.

Zhang Y, Klassen HJ, Tucker BA, Perez MT, Young MJ (2007) CNS progenitor cells promote a permissive environment for neurite outgrowth via a matrix metalloproteinase-2-dependent mechanism. J Neurosci 27:4499-4506.

Zhou Q, Wang S, Anderson DJ (2000) Identification of a novel family of oligodendrocyte lineage-specific basic helix-loop-helix transcription factors. Neuron 25:331-343. 